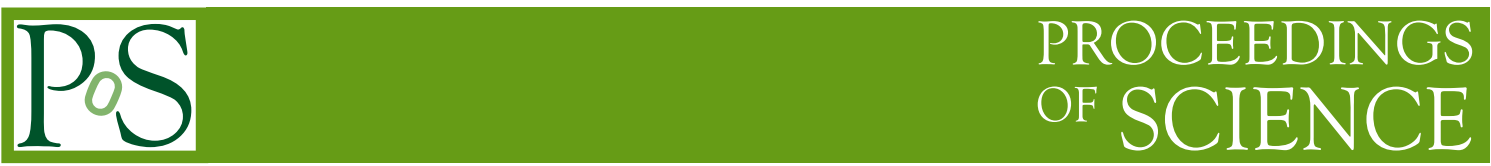

\title{
Galactic contribution to the IceCube astrophysical neutrino signal
}

\author{
Andrii Neronov* \\ University of Geneva \\ E-mail: andrii.neronoveunige.ch
}

\section{Dmitry Semikoz}

APC, Université Paris VII

\begin{abstract}
We use Fermi $\gamma$-ray and IceCube neutrino data to demonstrate that the IceCube astrophysical neutrino signal has significant contribution from the Milky Way galaxy. The astrophysical neutrino spectrum above $10 \mathrm{TeV}$ is found to lie at the high-energy extrapolation of the $\gamma$-ray spectrum below several TeV. The combined spectrum is well described by a pion decay emission from a parent Galactic cosmic ray population with a powerlaw spectrum with the slope $p \simeq 2.45$. In the energy band above $100 \mathrm{TeV}$, where the astrophysical neutrino signal is background-free, the Galactic latitude distribution of the neutrino signal reveals an evidence for significant Galactic component of the flux. The average Galactic cosmic ray spectrum derived from the combined $\gamma$-ray and neutrino data is consistent with a theoretical model of cosmic ray injection by diffusive shock acceleration followed by escape through the Galactic magnetic field with Kolmogorov turbulence. The locally observed $\mathrm{TeV}-\mathrm{PeV}$ cosmic ray proton spectrum is softer than the average Galactic cosmic ray spectrum. This could be readily explained by variability of injection of cosmic rays in the local interstellar medium over the past $10^{7} \mathrm{yr}$ and discreetness of the cosmic ray source distribution.
\end{abstract}

The 34th International Cosmic Ray Conference,

30 July- 6 August, 2015

The Hague, The Netherlands

\footnotetext{
* Speaker.
} 


\section{Introduction}

Precision measurements of the properties of the cosmic ray flux collected by a variety of detectors provide data only at a single location within the Galaxy: the position of the Solar system. It is not obvious if these measurements are representative of the average properties of the cosmic ray population in the Galaxy. The locally measured properties of the comic ray flux could be also determined by the peculiarities of recent injection of particles in the local interstellar medium $[1,2,3,4,5,6]$. Recent the data on cosmic ray positrons and antiprotons and also the slope and anisotropy properties of the cosmic ray nuclei spectra suggest that the $\mathrm{TeV}$ range cosmic ray spectrum has a strong contribution a supernova which exploded approximately two million years ago within one-two hundred parsec distance from the Sun [5, 6]. The data on deposition of isotopes in the deep ocean crust provide information on the occurrence of such nearby supernova events [7] indicate that only one such nearby supernova event has occurred over last 14 million years $[8,9]$. The slope of the locally observed cosmic ray spectrum in the $\mathrm{TeV}$ range might be largely determined by this recent supernova event. The information on the "characteristic" slope of the Galactic cosmic ray spectrum is completely erased by this last supernova contribution.

Complementary information on the cosmic ray source and propagation parameters is provided by secondary $\gamma$-rays and neutrinos. Contrary to the charged cosmic rays, neutral $\gamma$-rays and neutrinos go straight from their production sites to the Earth. $\gamma$-ray and neutrino signal from individual sources could provide information on the injection spectrum of cosmic rays, while diffuse $\gamma$-ray and neutrino emission from the ISM could provide the data on the propagation of cosmic rays in the Galaxy. Analysis of the $\gamma$-ray signal from the Galactic Plane of the Milky Way Galaxy and from the disk of the Large Magellanic Cloud (LMC) galaxy suggest that the average slope of the cosmic ray spectra produced by the star formation in these two galaxies is $p \simeq 2.45$, i.e. harder than that of the locally measured cosmic ray spectrum $[10,11]$. The slope of the neutral pion decay $\gamma$-ray spectrum produced by the interactions of cosmic rays with such hard slope is about $p_{\gamma} \simeq 2.4$.

An independent verification of the result on the hard $p \simeq 2.45$ average cosmic ray spectrum resulting from the star formation in the Milky Way and other galaxies could be obtained through the neutrino channel. The slope of the spectrum of neutrino emission from the Milky Way and other star forming galaxies is expected be about $p_{v} \simeq 2.4$, close to the slope of the $\gamma$-ray spectrum. The IceCube collaboration has recently reported the detection of astrophysical neutrino signal in the energy range from $10 \mathrm{TeV}$ to $2 \mathrm{PeV}[12,13,14,15]$. The signal forms a powerlaw spectrum $d N_{v} / d E \propto[E / 100 \mathrm{TeV}]^{-p_{v}}$, with $p_{v}=2.46 \pm 0.12[15]$.

This slope and normalisation of the neutrino spectrum are obviously inconsistent with the possibility that the observed neutrino flux is produced by cosmic rays in the Milky Way and / or other star forming galaxies, if one assumes that the typical spectrum of the cosmic rays resulting from the star formation is about the locally observed cosmic ray spectrum slope, $p \simeq 2.7$, and it has a knee feature exactly at the same energy as the knee of the locally measured cosmic ray spectrum [16] (see also [17]). At the same time, the measured slope of the astrophysical neutrino spectrum is consistent with the neutral pion decay $\gamma$-ray spectrum of the Milky Way and LMC disks [10, 11], as expected if the typical slope of the cosmic ray spectrum resulting from the star formation is $p \lesssim 2.5$ rather than $p \simeq 2.7$. Below we show that not only the slope, but also the normalisation of the neutrino flux is consistent with this hypothesis [18]. We argue that this suggests the validity of 
a simple model in which the injection of cosmic rays with the spectrum with the slope suggested by the DSA, $p_{i n j} \simeq 2 \ldots 2.2$ is followed by the softening produced by the escape through the galactic magnetic fields with Kolmogorov turbulence spectrum.

\section{2. $\gamma$-ray and neutrino all sky spectra and anisotropies}

Our analysis uses all publicly available Fermi / Large Area Telescope (LAT) data collected over the period from August 2008 till June 2014. We have processed the data using the Fermi Science Tools $v 9 r 32 p 5^{1}$ - a standard software package, provided by the Fermi collaboration to reduce the data, obtained by the Fermi/LAT. We have used the Pass 7 "reprocessed" event selection.

We have filtered the event lists using the gtselect tool with parameter evclass $=3$, which leaves the $\gamma$-ray events and rejects most of the cosmic ray background events. To produce the allsky spectrum shown in Fig. 2, we have used the aperture photometry method ${ }^{2}$, applied to the full sky. An estimate of the exposure in each energy bin was done using the gtexposure tool with the option apcorr=no (there is no need to correct the exposure for the point spread function for the full sky). To separate the diffuse emission from the point source contributions we subtract the flux in the circles of the radius $0.5^{\circ}$ around point sources from the four-year Fermi catalogue [19]. This is sufficient for the analysis of the diffuse emission in the energy band above $10 \mathrm{GeV}$. In this energy band the point spread function of the LAT is smaller than $\sim 0.3^{\circ}$.

To produce the Galactic latitude profiles for the neutrino data, we have binned the neutrino events reported by [14] in Galactic latitude and have estimated the IceCube exposure in each Galactic latitude bin. This was done via a Monte-Carlo (MC) simulation of the Galactic latitude distribution of neutrino events driven from an isotropic sky distribution, with account of the declination dependence of the IceCube effective area, derived from the information reported by [14]. Having estimated the exposure in each Galactic latitude bin, we have multiplied the model fluxes of the Galactic and isotropic components by the Galactic latitude dependent exposure to estimate the expected number of neutrino counts in each latitude bin.

Fig. 1 shows the combined $\gamma$-ray and neutrino all-sky spectrum in a broad GeV-PeV energy range. One could see that the neutrino spectrum lies at the high-energy extrapolation of the $\gamma$-ray spectrum of the entire sky. Thus, not only the slopes, but also the normalisation of the two spectra agree with each other. The uncertainty of the neutrino flux at $100 \mathrm{TeV}$ is just by a factor of $\simeq 2$. Assuming a negligible uncertainty of the $\gamma$-ray flux at $\simeq 30 \mathrm{GeV}$, one could find that a powerlaw fit to the combined $\gamma$-ray plus neutrino spectrum gives a very precise measurement of the slope of the powerlaw, $p_{v \gamma}=2.37 \pm 0.05$, with an error $\Delta p_{\gamma v}=\log (2) /[2 \log (100 \mathrm{TeV} / 100 \mathrm{GeV})] \simeq 0.05$. This is due to a very large dynamic range of the energy on which the powerlaw is observed and to the moderate uncertainty of the neutrino flux measurement. Red and blue thin solid curves show a model of the neutrino and $\gamma$-ray emission from interactions of cosmic rays with the ISM. The cosmic rays have a powerlaw spectrum with the slope $p=2.45$. The $\gamma$-ray and neutrino spectra are calculated using the parametrizations of the pion production spectra by $[20,21]$. The model does

\footnotetext{
${ }^{1}$ http://fermi.gsfc.nasa.gov/ssc/data/analysis/

${ }^{2}$ http://fermi.gsfc.nasa.gov/ssc/data/analysis/scitools/

aperture_photometry.html
} 

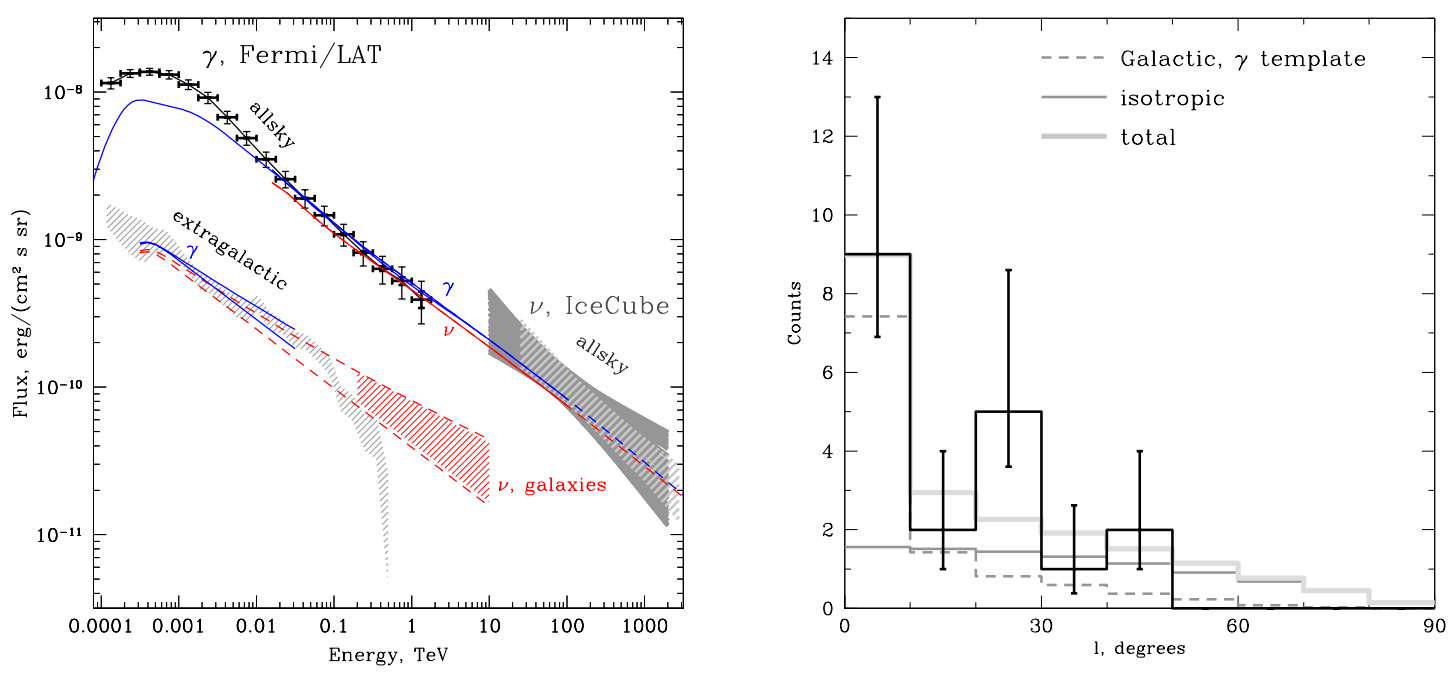

Figure 1: Left: Gamma-ray and neutrino spectra of the full sky. Red and blue thick solid curves show the neutrino and $\gamma$-ray emission from protons with the powerlaw spectrum with the slope $p=2.45$. Thin solid blue and dashed red curves show the estimates of possible $\gamma$-ray and neutrino fluxes from star forming galaxies. Dark grey shaded range shows the IceCube measurement from Ref. [15]. Light grey hatched range shows the 4-yr exposure update reported in Ref. [37]. Right: Galactic latitude distribution of neutrino events with energies above $100 \mathrm{TeV}$ (taking into account the update from the Ref. [37]). Grey histograms show a best fit model with nearly equal fluxes of Galactic (dashed) and extragalactic (thin solid) components contributing to the total (thick light grey) flux.

not fit the $\gamma$-ray data in the energy band below $\sim 10 \mathrm{GeV}$. This is expected, because in this energy band significant contribution from electron Bremsstrahlung is expected [22, 10].

The neutrino flux from star forming galaxies other than the Milky Way is constrained by the measurement of an upper limit on the $\gamma$-ray flux of star forming galaxies, which is given by the measured isotropic $\gamma$-ray background (IGRB) flux [23]. Assuming that the $\gamma$-ray emission from cosmic ray interactions in star forming galaxies saturates the IGRB measurement, one could estimate the neutrino flux from star forming galaxies via extrapolation of the IGRB spectrum toward higher energies. This gives an upper limit of $\sim 50 \%$ of the contribution to the IceCube signal.

If a large part of the astrophysical neutrino signal is of Galactic origin, one expects to find higher signal level at low Galactic latitudes. The neutrino signal indeed shows a hint of anisotropy in the direction of the Galactic Plane [14], which is consistent with the $\gamma$-ray - neutrino signal correlation. The distribution of neutrinos along the Galactic Plane is consistent with the distribution of the $\gamma$-rays, with higher event statistics observed around the region of Galactic Ridge [14, 24].

To find the anisotropy properties of the Galactic component of the neutrino flux, we use the observed Galactic latitude profile of the $\gamma$-ray emission in the energy band above $300 \mathrm{GeV}$ as a template [18]. This is possible because Fig. 1 suggests that the $\gamma$-ray and neutrino signals are both of hadronic origin and the neutrino signal above $10 \mathrm{TeV}$ could be directly calculated from the $\gamma$-ray signal via s simple powerlaw extrapolation. In the energy band above $300 \mathrm{GeV}$ the $\gamma$-ray signal is free from the extragalactic contribution which is suppressed by the effect of gamma-gamma pair 
production on the Extragalactic Background Light [23]. Thus, the neutrino signal above $10 \mathrm{TeV}$ is a sum of the anisotropic Galactic component which follows the $\gamma$-ray anisotropy template plus an isotropic extragalactic component.

The right panel of Fig. 1 shows the distribution of the neutrino events with energies above $100 \mathrm{TeV}$ in Galactic latitude. In this energy band, the neutrino signal is almost free from the residual atmospheric neutrino and muon backgrounds $[14,37]$. The grey solid histogram shown in the same panel presents the expected Galactic latitude distribution of the signal expected if the neutrino signal is isotropic (i.e. is exclusively extragalactic origin). The isotropic model distribution is calculated using the MC simulations described above [18]. Dashed histogram shows the expected profile of the neutrino signal from the Galaxy, calculated based on the $\gamma$-ray template as explained above.

From this figure one could see that if the Galactic component of the flux would be absent, the isotropic flux model alone would be at tension with the data. In this case the isotropic flux wold have to be normalised at the total number of counts in the data. This would increase the normalisation by a factor of 2 compared to the thin solid histogram shown in the figure. In the data there are no detected events in the Galactic latitude bins with $|b| \geq 50^{\circ}$. At the same time, the isotropic-only model predicts 4.6 events. The MC simulations show that the probability to find this in the data sets of 19 events derived from the isotropic flux is $p_{|b|>50^{\circ}}=5 \times 10^{-3}$. In addition, the predicted number of events in the first bin $|b|<10^{\circ}$ is 3.2 for the isotropic-only model, while the number of detected events is 9 . This is inconsistent with the model at the probability level $p_{|b|<10^{\circ}}=2 \times 10^{-3}$. The overall probability of the combined over-prediction of event statistics at high Galactic latitudes and under-prediction at low Galactic latitudes is $p=7 \times 10^{-5}$, which is equivalent to $\mathrm{a} \simeq 4 \sigma$ deviation of the model from the data in terms of an equivalent Gaussian statistics signal.

The above conclusion about the $4 \sigma$ inconsistency of the isotropic model with the data is a "post-trial" conclusion. To have a fair judgement of significance of this inconsistency, one needs to take into account the trial factor related to the choice of the Galactic latitude bins where the inconsistency of the model with the data is largest. There is no trial factor to be taken into account for the general choice of the pattern "high Galactic Latitude \& low Galactic latitude" bins $|b|>b_{\text {min }}$ and $|b|<10^{\circ}$, because these are the bins which contains the lowest and the highest would-be Galactic signal. If the data are inconsistent with the isotropic model because of the presence of Galactic component, these are the bins where the inconsistency is expected to appear at the first place. The only trial factor to take into account is in the choice of $b_{\min }$. Putting $b_{\min } \rightarrow 90^{\circ}$ (i.e. completely ignoring the deficit at high Galactic latitudes) decreases the significance of the inconsistency of the isotropic model with the data down to $3 \sigma$ level.

From the right panel of Fig. 1 one could see that addition of the Galactic contribution to the neutrino flux removes the tension between the model and the data. Most of the counts in the bin $|b|<10^{\circ}$ could be readily attributed to the Galactic component. The Galactic flux normalised on $|b|<10^{\circ}$ bin is expected to produce only 0.4 counts in the $|b|>50^{\circ}$ bin. The Galactic-only model deviates from the data by 2.2 sigma (probability $2.7 \times 10^{-2}$, because of the over-abundance of events in the $20^{\circ}<|b|<30^{\circ}$ bin ( 5 at expected $\simeq 2$ ).

The best fit Galactic plus isotropic model has approximately equal contributions from the two components in the overall flux. Precise measurement of the relative normalisation of the Galactic 
and isotropic contributions to the $E>100 \mathrm{TeV}$ neutrino flux is not possible, due to the low statistics of the signal. However, discrimination between the dominant Galactic or dominant extragalactic flux models will be possible already with a deeper IceCube in its present configuration and also with more powerful detectors like IceCube-Gen2 [25] or km3net [26].

At the first sight, this result seems to be in slight tension with the analysis of [17] who finds that the Galactic diffuse emission could not contribute more than $50 \%$ of the observed neutrino signal. However, the discrepancy stems from model dependence of the results. One could notice that the result of [17] depends on the choice of the spatial template for the hadronic component of the all-sky $\gamma$-ray flux. It relies on a spatial template derived by the Fermi collaboration [22] assuming the reference cosmic ray spectrum with the slope 2.7 (rather than 2.58 assumed by [17]). The uncertainty of this template and its dependence on the assumed spectral shape of the template are difficult to estimate. In our analysis we assume that the Galactic longitude profile of the Galactic component of neutrino flux is identical to the profile of the $\gamma$-ray emission in the energy band above $300 \mathrm{GeV}$. This is motivated by the fact that the isotropic and the inverse Compton components of the $\gamma$-ray flux are suppressed at these energies. In such a way in our analysis we avoid using a model-dependent spatial template of the Galactic component of neutrino flux.

Detection of neutrino and $\gamma$-ray emission from the Galactic cosmic rays with $p=2.45$ powerlaw index provides an important clue for understanding of formation of Galactic cosmic ray spectrum $[5,10]$. It is consistent with the possibility that the cosmic rays are injected with the powerlaw spectrum with the slope $p=2.1 \ldots 2.2$ predicted by the shock acceleration models [32, 33, 34], with the energy dependence of the diffusion coefficient $D(E) \sim E^{1 / 3}$ as expected for the Kolmogorov turbulence spectrum of the ISM [27].

Although the slope and normalisation of the Galactic neutrino spectrum are fixed by the $\gamma$ ray data at lower energies, there is no certainty about its high-energy end. The Galactic cosmic ray spectrum should have a knee like suppression at high energies produced by the escape from the Galactic Disk and / or high-energy cut-off due to the absence of sources capable of particle acceleration beyond certain energy range $[28,29,30]$. Similarly to the slope of the spectrum, the energy of the knee measured in the local Galaxy is not necessary the same as the average knee energy for the Galactic cosmic rays. The knee feature could be explained within the escape model of formation of cosmic ray spectrum as the energy at which the scattering length of cosmic rays becomes comparable to the correlation length of the Galactic magnetic field [31]. This model fits the shapes of the spectra of all elemental groups around the knee energy [29, 30]. Within the escape model, the knee energy varies across the Galaxy and from one galaxy to another in response to the variations of the properties of the galactic magnetic field. Presence of the knee of the cosmic ray spectrum leads to a suppression of the neutrino flux at the energies $\sim 0.05$ of the knee energy. Uncertainty of the average position of the knee in the Galactic cosmic ray spectrum introduces an uncertainty in the shape of the spectra of Galactic $\gamma$-ray and neutrino emission in the PeV energy band (the dashed model line ranges in Fig. 1).

The locally observed $\mathrm{TeV}-\mathrm{PeV}$ cosmic ray proton spectrum appears "peculiar" in the sense that its slope is different from the slope of the average Galactic cosmic ray spectrum. This peculiarity could not be related to the specific of the propagation process in the local ISM, because in this case also the spectra of atomic nuclei would be affected. It could also hardly be related to the process of injection from the Galactic cosmic ray sources in general, because in this case the proton 
spectrum would be systematically softer than the nuclei spectrum everywhere in the Galaxy and this would be visible in the softer $\gamma$-ray / neutrino spectrum of the Galaxy.

The most probable reason for the peculiarity of the locally observed proton spectrum is in the change of the cosmic ray injection rate over the last $T \sim 10^{7}$ yr $[3,4,5,6]$. Massive injection of cosmic rays in the local ISM in a star formation episode $10^{7} \mathrm{yr}$ ago could have resulted in an increase of the cosmic ray energy density at all energies. Faster diffusion of higher energy particles should have led to faster "wash out" of the excess density at high-energies and, as a consequence, to a temporary softening of the local cosmic ray spectrum. Several candidate past events in the local Galaxy, like e.g. the event which produced an expanding ring of molecular clouds, the Gould Belt $[35,36]$, could be considered. In a similar way, a nearby supernova explosion which occurred several million years ago could have produced an additional distortion in the cosmic ray spectrum. Evidence for such an event could be found through the analysis of the secondary positrons and antiprotons in the cosmic ray spectrum [5] and anisotropy [6]. Discreteness of the distribution of the cosmic ray sources and time variability of the star formation rate generically leads to a situation in which the locally measured cosmic ray spectrum is not identical to the average Galactic cosmic ray spectrum $[5,6,10]$.

\section{Conclusions}

We have shown that the the all-sky neutrino spectrum in the TeV-PeV energy range coincides with the high-energy extrapolation of the all-sky $\gamma$-ray spectrum in the energy band below $1 \mathrm{TeV}$. Such a consistency of the $\gamma$-ray and neutrino spectra is not unexpected in a simple model where both $\gamma$-rays and neutrinos originate in the decays of neutral and charged pions produced by interactions of cosmic rays in the Milky Way and other galaxies.

Noticing that the $\gamma$-ray all-sky spectrum in the energy band just below $1 \mathrm{TeV}$ is dominated by the Galactic contribution, we were able to estimate the flux and anisotropy properties of the Galactic component of the neutrino spectrum. The isotropic neutrino flux from the cosmic ray interactions in star forming galaxies other than Milky Way is constrained by the level of the IGRB and could not constitute more than $\sim 50 \%$ of the observed neutrino flux. Presence of significant Galactic component of the neutrino flux (at least $50 \%$ of the flux) is also consistent with the anisotropy properties of the neutrino signal. We find that the isotropic-only model of neutrino flux above $100 \mathrm{TeV}$ is inconsistent with the IceCube data at $\gtrsim 3 \sigma$ level.

Combination of the $\gamma$-ray and neutrino data suggests that the Galactic cosmic ray spectrum is harder than previously thought, with the slope $p \simeq 2.5$ in the $\mathrm{TeV}-\mathrm{PeV}$ energy range. The discrepancy between the slopes of the average Galactic cosmic ray spectrum and the locally measured cosmic ray spectrum is readily explained by the discreteness of the cosmic ray source distribution and time variability of the local star formation rate.

\section{References}

[1] Ptuskin V.S., Jones F.C., Seo E.S., Sina R., Ad.Sp.Res. 37, 1909 (2006).

[2] Ahn H.S., et al., Ap.J., 714, L89 (2010).

[3] Neronov A., Semikoz D.V., Phys. Rev., D85, 083008 (2012). 
[4] Eicher D., Kumar R., Pohl M., Ap.J., 769, 138 (2013).

[5] Kachelriess M., Neronov A., Semikoz D., arXiv:1504.06472 (2015).

[6] Savchenko V., Kachelriess M., Semikoz D.V., arXiv:1505.02720.

[7] Ellis J., Fields B.D., Schramm D.N., Ap.J., 470, 1227 (1996).

[8] Fields B.D., Hochmuth K.A., Ellis J., Ap.J., 6210, 902 (2005).

[9] Fry H.J, Fields B.D., Ellis J., Ap.J., 800, 71 (2015).

[10] Neronov A., Malyshev D., arXiv:1505.07601 (2015).

[11] Foreman G., Chu Y.-H., Gruendl R., Hughes A., Fields, B., Ricker P., arXiv:1502.04337 (2015).

[12] IceCube Collaboration, Science, 342, 947 (2013).

[13] Aartsen M.G. et al., Phys. Rev. Lett., 111, 021103 (2013).

[14] Aartsen M.G. et al. [IceCube Collaboration], Phys.Rev.Lett., 113, 101101 (2014).

[15] Aarsten M.G. et al., arXiv:1410:1749 (2014).

[16] M. Kachelriess and S. Ostapchenko, Phys. Rev. D 90, 083002 (2014).

[17] Ahlers M., et al., arXiv:1505.03156 (2015).

[18] A.Neronov, D.V.Semikoz, Astroparticle Physics (2015) [arXiv:1412.1690].

[19] Fermi Collab., arXiv:1501.02003 (2015).

[20] Kelner S.R., Aharonian F.A., Bugayov V.V., Phys.Rev., D74, 034018 (2006).

[21] Kamae T., Karlsson N., Mizuno T., Abe, T., Koi T., Ap.J., 647, 692 (2006).

[22] Ackermann M., et al., Ap.J., 750, 3 (2012).

[23] The Fermi LAT collab., Ap.J., 79986 (2015).

[24] Neronov A., Semikoz D., Tchernin C., Phys.Rev., D89, 103002 (2014).

[25] Aarsten M.G., et al., arXiv:1412.5106 (2014).

[26] Bagley P. et al., km3net TDR http://www.km3net.org/TDR/TDRKM3NeT.pdf (2009).

[27] Armstrong J.W., Rickett B.J., Spangler S.R., Ap.J., 443, 209 (1995).

[28] Berezinskii V.S., et al. The Astrophysics of Cosmic Rays, Nothr-Holland, Amsterdam (1990).

[29] Giacinti G., Kachelriess M., Semikoz D.V., Phys.Rev. D90, 041302 (2014).

[30] Giacinti G., Kachelriess M., Semikoz D.V., Phys.Rev. D91, 083009 (2015).

[31] Ginzburg V.L., Syrovatskii S.I., The Origin of Cosmic Rays (Pergamon Press, Oxford, 1964).

[32] Krymskii G.F., Doklady Akademii Nauk SSSR, 234, 1306 (1977).

[33] Bell A.R., Royal Astronomical Society, 182, 147 (1978)

[34] Malkov M., Drury L.O.C. Rep. Progr. Phys. 64, 429 (2001).

[35] Gehlers N., et al., Nature, 404, 363 (2000).

[36] Neronov A., Semikoz D.V., Taylor A.M., Phys. Rev.Lett., 108, 051105 (2012).

[37] O.Bottner, talk at IPA 2015 Symposium, http://meetings.wipac.wisc.edu/IPA2015/home (2015). 